\title{
Silvia Camilotti
}

\section{Anilda Ibrahimi, Non c’è dolcezza, Torino, Einaudi, 2012.}

\begin{abstract}
Si propone una recensione del romanzo Non c'è dolcezza (Einaudi 2012) di Anilda Ibrahimi, scrittrice di origine albanese, residente in Italia e autrice in lingua italiana.

Nei suoi precedenti romanzi - Rosso come una sposa (Einaudi 2008) e L'amore e gli stracci del tempo (Einaudi 2009) - l'autrice pone l'attenzione sul conflitto balcanico e sulla storia albanese. Uno dei temi privilegiati nella sua scrittura è la condizione femminile nel suo paese natale: nei suoi romanzi offre un'immagine anticonvenzionale di donne forti che, nonostante la cultura tendenzialmente patriarcale, rappresentano sempre i pilastri della famiglia.

Ibrahimi non alimenta una visione stereotipata di donna succube, che troppo spesso i lettori italiani possiedono. L'attenzione alle questioni di genere, ai rapporti tra uomini e donne e ai condizionamenti sociali e culturali è centrale nei suoi testi.
\end{abstract}

Anilda Ibrahimi, scrittrice di origine albanese e residente in Italia dal 1997, ritorna con Non c'è dolcezza su temi affrontati nei due testi precedenti, Rosso come una sposa e L'amore e gli stracci del tempo, entrambi usciti per i tipi Einaudi: l'attenzione per la storia albanese e balcanica e la focalizzazione sul punto di vista femminile.

L'opera di Ibrahimi si colloca all'interno di quella branca della letteratura italiana definita "letteratura della migrazione" che include le opere composte in lingua italiana, senza la mediazione della traduzione, da autori e autrici immigrati in Italia. Si tratta di un fenomeno letterario di poco più d'una ventina d'anni, che, seppure in un arco di tempo relativamente limitato, ha mostrato e sta tuttora mostrando una grande vivacità e interessanti esiti di scrittura. Una categoria come quella di "letteratura della migrazione" può risultare semplificante, oramai, per molti degli autori e delle autrici che, in virtù della loro esperienza di vita e scrittura, rientrerebbero a pieno titolo allinterno di tale etichetta, che tuttavia viene superata dall'evoluzione in termini di forma e contenuto della loro opera. È questo il caso di Ibrahimi, i cui romanzi si emancipano dalle forme dell'autobiografia e del diario e dall'uso di una lingua non sempre affinato, come accadeva all'inizio degli anni Novanta con i primi testi

The e-journal «altrelettere» is hosted at the URL: http://www.altrelettere.uzh.ch , in accordance with the Open Access Policy of the University of Zurich. Please cite this article as follows: Silvia CAMILOTTI, Recensione a Anilda Ibrahimi, "Non c’è dolcezza”, Torino, Einaudi, 2012, in «altrelettere», 10.9.2012, DOI: 10.5903/al_uzh-9.

(C) This article is licensed under a Creative Commons Attribution 2.5. Switzerland (CC BY-NC-ND 2.5). Please read the license terms on the website: http://creativecommons.org/licenses/by-nc-nd/2.5/ch/deed.en 
di autori migranti. Il fatto stesso che le sue opere, all'interno della produzione Einaudi, non appartengano a una particolare collana dedicata ma rientrino tra quelle di scrittori italiani contemporanei è ulteriore conferma di tale sorta di riconoscimento, che considera i romanzi dell'autrice parte della letteratura italiana tout court.

Inoltre, la grande sensibilità di Ibrahimi sulla condizione femminile e sui rapporti di gender rende la sua opera ulteriormente interessante, poiché oltre ad aprire delle finestre sul mondo dei Balcani accende i riflettori sulle donne, decostruendo una serie di stereotipi fuorvianti. Tale attenzione pare infatti attraversare sin dagli esordi la sua scrittura, ${ }^{1}$ che ha sempre visto ambientazioni albanesi (in particolare Rosso come una sposa e Non c'è dolcezza) o comunque situate in area balcanica (si pensi a L'amore e gli stracci del tempo che si snoda sullo sfondo dell'ultimo conflitto balcanico). Emerge dunque la volontà di raccontare le vicende - inserendole in una cornice storica di ampio respiro che abbraccia quasi tutto il ventesimo secolo - degli abitanti di questi territori, così vicini all'Italia ma marginali e per molti versi dimenticati. Il tentativo di mostrare da una parte la condizione difficoltosa di molte donne, soprattutto nelle aree rurali, e dall'altra la loro determinazione e la solo superficiale subalternità nelle relazioni di gender contribuisce a rendere più articolata e complessa l'immagine, spesso inferiorizzante, delle stesse.

Non c'è dolcezza è un romanzo che pone al centro i temi della maternità e della complicità tra donne: Lila e Eleni sono amiche da sempre, cresciute in un paesino dell'entroterra albanese che assiste alle vicende storiche che scuotono il paese nella seconda metà del Novecento: spicca a tale proposito la continuità con i romanzi precedenti di Ibrahimi, in particolare Rosso come una sposa, in cui l'autrice colloca in un contesto ampio, quasi epico, storie di uomini e donne le cui vite sono inevitabilmente intrecciate e condizionate dalla Storia.

Lila, in nome della promessa fatta all'amica, dona, a lei che non riesce ad avere figli, questo bambino, inconsapevole delle conseguenze che una scelta simile può provocare. Sarà una perdita che la donna non riuscirà ad accettare e che segnerà la svolta verso il suo declino. Il primo a subirne le conseguenze è il figlio stesso, 
Arlind, che vive una sensazione inspiegabile di incompletezza, nonostante quelli che ritiene i suoi genitori lo ricoprano di attenzioni. Egli cresce convinto di essere figlio di Eleni e Andrea, anche se il rapporto con quella che considera zia Lila resta inspiegabilmente, ai suoi occhi, viscerale. Sono gli occhi di Arlind che rimarcano un vuoto di cui non riesce a darsi ragione, che non prevedono dolcezza, come recita anche il titolo del romanzo:

Arlind ha sempre vissuto in attesa di qualcosa. Gli sembrava che gli mancasse sempre un pezzo, in ogni momento. Guardava la grande torta che sua madre gli aveva preparato per il compleanno e pensava che mancasse un ingrediente che l'avrebbe resa migliore e così non gli andava più di mangiarla. E anche la tazza di latte che gli portava Eleni al mattino era sempre insufficiente, mancava il resto rimasto nella pentola. Ma poi non riusciva nemmeno a finirla e ci immergeva il gatto. Tutti dicevano che era viziato, ma lui non si sentiva viziato. Piuttosto, incompleto (IBRAHIMI, 209).

Tale sensazione si placa solo all'altezza dell'epilogo, quando la verità verrà a galla, anche se accompagnata da una serie di nuove domande. La morte di Lila produce infatti strane conseguenze, non ultima la scomparsa di suo marito, padre naturale di Arlind. In tal caso, i radicali cambiamenti politici sembrano avere un ruolo in questa misteriosa vicenda, riportando su un piano nazionale le vicende individuali. La Storia ritorna a influenzare le vite dei personaggi, in una continua altalena tra personale e politico che caratterizza tutti i romanzi di Ibrahimi.

L'intera vicenda permette di riflettere su questioni quali l'identità, la fedeltà tra donne ed i rapporti di gender. La lettura di queste pagine induce chi legge a chiedersi infatti quanto i legami di sangue possano essere rescissi e sostituiti con altri, quanto il rapporto tra madre e figlio possa venire accantonato in nome di una promessa tra amiche, in che misura un bambino e la sua madre naturale risentano di una privazione che nasceva da una decisione consapevole, dettata da un profondo legame di amicizia tra donne. Chi legge vede Lila ed Eleni crescere insieme, superare la contrarietà dei genitori (Eleni appartiene a una famiglia povera, a differenza di Lila), invaghirsi dello stesso ragazzo, ma non per questo divenire mai avversarie. Sarà il mantenimento della promessa a gettare un'ombra sul loro rapporto. Tra i tre romanzi di Ibrahimi, è quest'ultimo a incentrarsi in 
particolare sul rapporto d'amicizia esclusivo tra due donne; in Rosso come una sposa, la protagonista Saba non trova una simile complicità con un'amica e nel testo si dà maggior spazio ai rapporti - non sempre distesi - interni a più generazioni della stessa famiglia allargata. Ne L'amore e gli stracci del tempo la focalizzazione riguarda il rapporto tra Zlatan e Ajkuna, che crescono insieme e che la guerra dei Balcani dividerà.

In questo romanzo l'attenzione va specificamente al rapporto femminile che supera le gerarchie imposte socialmente secondo cui non è bene che una giovane di buona famiglia si accompagni ad una di più misera condizione. Ma non saranno regole simili a dividere Lila e Eleni, bensì l'impossibilità della prima di accettare di aver donato suo figlio all'amica: nella società di allora, una coppia priva di figli non era ben accolta, e la compassione di Lila l'ha spinta a privarsi del suo ultimo figlio, credendo che un legame filiale potesse facilmente spezzarsi in nome dell'amicizia tra donne e della tutela dell'immagine pubblica dell'amica.

Eleni e Lila sono personagge ${ }^{2}$ a tutto tondo, con le debolezze e la forza di molte delle donne di Ibrahimi e, in tal senso, profondamente umane. In continuità con i romanzi precedenti, i personaggi femminili non appaiono affatto succubi e passivi, ma motori delle vicende e capaci di imporsi, anche sugli uomini. Il marito di Lila accetta, seppure con delle iniziali reticenze, la decisione della moglie di cedere il loro ultimo nato: la descrizione del loro rapporto di coppia smantella il luogo comune che vede nell'uomo colui che decide per conto della moglie. Da un punto di vista socio-culturale, poi, il fatto che egli accetti di donare l'unico figlio maschio è indice di grande sensibilità e rispetto nei confronti delle decisioni della amata moglie, il cui parere è più forte di certe tradizioni patriarcali. Tale esempio scardina il luogo comune della subalternità femminile, che Ibrahimi non avvalla mai, in alcuno dei suoi testi: ciò non significa negare la fatica che in molti frangenti l'essere donna implica, soprattutto nelle aree rurali (l'esperienza di Lila che lavora e vive in città è infatti diversa da quella di Eleni che resta in un contesto più marginale) ma implica la valorizzazione della saggezza e della agency femminile che si misura nella capacità di ritagliarsi spazi di autonomia e decisione e di coltivare aspettative di riscatto. 
In tal senso la rappresentazione che Ibrahimi offre dei rapporti di gender è molto più articolata e non si limita all'assunto che considera la società albanese $\mathrm{o}$ più ampiamente balcanica arretrata se osservata da tale punto di vista: senza negare la chiusura che il paese delle aquile ha a lungo sofferto e le restrizioni nei confronti delle donne, Ibrahimi valorizza la determinazione, l'ambizione e la forza di volontà di molte donne. Ciò emerge in particolare nelle dinamiche di coppia: abbiamo già fatto cenno al rapporto tra Lila e suo marito, ma anche le relazioni di coppie più marginali alla vicenda confermano il ruolo decisivo delle donne nella gestione della relazione. Il parere delle anziane, poi, è ricercato e rispettato: nei romanzi di Ibrahimi, incluso quest'ultimo, esse vengono ritratte con grande benevolenza e la loro saggezza le rende dei punti di riferimento.

Tale rappresentazione inedita, che valorizza il ruolo femminile e la forza di volontà delle personagge, depotenzia il rischio di eurocentrismo latente in tanta letteratura che pone al centro culture e popoli considerati dal mainstream "marginali": il metro di giudizio secondo cui certe tradizioni culturali vengono considerate arretrate spesso guarda alla condizione femminile. In un paese come l'Albania - anche se, come Ibrahimi mostra, all'interno dello stesso paese vi sono contesti diversificati - lo squilibrio nei rapporti di gender è forse meno profondo di quanto sembri e una tradizione che nell'immaginario "occidentale" è patriarcale e maschilista lascia alle donne spazi di agency maggiori di quanto si pensi: le figure di Lila e Eleni lo dimostrano, ma anche l'importanza che le anziane rivestono ne è un ulteriore indicatore.

Ibrahimi non alimenta in alcun modo tali vulgate eurocentriche e offre la fotografia di un paese che ha subito vorticosi cambiamenti in pochi decenni, un paese estremamente eterogeneo (città e campagna, zone costiere e montagne condizionano inevitabilmente modi e abitudini dei loro abitanti, offrendo a chi legge una versione complessa e non appiattita su un unico paradigma), in cui le donne sono protagoniste a tutti gli effetti.

Un'immagine molto bella che fa capolino in alcune pagine, per restare nel tema della rappresentazione anticonvenzionale, riguarda il ruolo che ha il popolo rom in Non c'è dolcezza. Il romanzo si apre con la loro carovana in arrivo al 
villaggio, accompagnata da canti e accolta festosamente dagli abitanti del paese; anche le loro vite, tuttavia, non potranno sottrarsi ai cambiamenti della Storia, che li renderanno vittime di politiche di discriminazione e repressione destinate a segnarli indelebilmente.

Note

1 Due recensioni ai romanzi sono reperibili ai seguenti link:

http://www.ilgiocodeglispecchi.org/libri/scheda/lamore-e-gli-stracci-del-tempo e

http://www.ilgiocodeglispecchi.org/libri/scheda/rosso-come-una-sposa

2 Il termine nasce dalle iniziative a cura della Società Italiana delle Letterate:

http://www.societadelleletterate.it/personagge/ 